\title{
An effective method for the detection of trace species demonstrated using the MetOp Infrared Atmospheric Sounding Interferometer
}

\author{
J. C. Walker, A. Dudhia, and E. Carboni \\ Atmospheric, Oceanic and Planetary Physics, Clarendon Laboratory, Parks Road, Oxford, OX1 3PU, UK \\ Received: 13 October 2010 - Published in Atmos. Meas. Tech. Discuss.: 26 October 2010 \\ Revised: 28 April 2011 - Accepted: 29 April 2011 - Published: 3 August 2011
}

\begin{abstract}
Fast and reliable methods for the detection of atmospheric trace species are needed for near-real-time applications including volcanic hazard avoidance. One common approach using hyperspectral instruments is to measure the difference in brightness temperature between a small number of target sensitive and background channels to determine the presence of the target species. Although fast and robust, current brightness temperature difference methods do not fully exploit the spectral range and resolution of hyperspectral instruments, and the noise associated with the measurements remains high. In this paper, we describe a way to make full use of the spectral information from hyperspectral sounders allowing the presence of the target species to be determined with much better sensitivity in near-real-time if required. The technique is demonstrated using the MetOp Infrared Atmospheric Sounding Interferometer considering two case studies: (a) the detection of sulphur dioxide from the eruption of the Kasatochi volcano in Alaska in August 2008, and (b) the detection of ammonia emissions related to agriculture over Southern Asia in May 2008. The performance of this method is compared against that of existing brightness temperature difference methods. It is found that the sensitivity of the detection of these trace species is improved by up to an order of magnitude.
\end{abstract}

\section{Introduction}

The Infrared Atmospheric Sounding Interferometer (IASI) on board the EUMETSAT MetOp platform is a Michelsontype Fourier transform spectrometer with continuous coverage in the thermal infrared from $645-2760 \mathrm{~cm}^{-1}$ sampled at a spectral resolution of $0.25 \mathrm{~cm}^{-1}$. The instrument

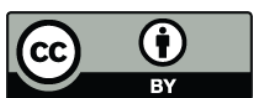

Correspondence to: J. C. Walker (walker@atm.ox.ac.uk) sits in a polar orbit with a footprint consisting of 4 circular instantaneous field-of-views each with a radius of $12.3 \mathrm{~km}$ at nadir which are scanned in the direction perpendicular to the line of flight with a swath width of about $2200 \mathrm{~km}$ giving global coverage on a daily basis. The spectral range and resolution coupled with excellent geographical coverage mean that the instrument is suitable for monitoring atmospheric composition. However, fully quantitative retrievals are slow and inefficient when searching for a sporadic event within a large dataset due to the computationally intensive radiative transfer calculations required. Faster methods are therefore needed to find such events quickly and easily, especially for near-real-time applications such as volcanic hazard avoidance.

A commonly used method for the fast detection of trace atmospheric species is to calculate the difference in brightness temperature (BT) between a small number of channels, some of which are sensitive to the target species and others of which are insensitive, carefully chosen so that the spectral background is well matched between channels so that it can be eliminated isolating the contribution of the target species. The difference in brightness temperature between channels is then compared against a threshold which indicates a positive detection. Such techniques have useful practical applications in hazard avoidance. Simple brightness temperature difference (BTD) filters for the detection of sulphur dioxide $\left(\mathrm{SO}_{2}\right)$, which is used as a proxy for volcanic ash which is dangerous to aviation and is generally more difficult to discriminate, are applied operationally to provide an early warning of volcanic activity and to help track the volcanic plume in near-real-time (Rix et al., 2009; van Geffen et al., 2009; Prata, 2009). Sulphur dioxide can be tracked from IASI using the difference in signal between two channels located at 1371.50 and $1371.75 \mathrm{~cm}^{-1}$ in the $\mathrm{SO}_{2} \nu_{3}$ vibrational band and two background channels at 1407.25 and $1408.75 \mathrm{~cm}^{-1}$ outside the $\mathrm{SO}_{2}$ band (Clarisse et al., 2008). Similarly, $\mathrm{SO}_{2}$ can be tracked from the Atmospheric Infrared

Published by Copernicus Publications on behalf of the European Geosciences Union. 
Sounder (AIRS) on board Aqua using a three channel method (OMI Volcanic Emissions Group TOMS, 2010). The four channel BTD filter for IASI is currently used to alert authorities within Europe who monitor the threat posed to aviation by volcanic ash clouds (Rix et al., 2009; van Geffen et al., 2009), with similar schemes in place using the AIRSAqua filters to alert authorities in the United States (OMI Volcanic Emissions Group TOMS, 2010). The main drawback of these techniques, however, is that they do not make full use of the wide spectral range and fine spectral resolution available from hyperspectral instruments, using only a small fraction of the channels which could provide useful information. Although sensitivity is adequate for many events, significant eruptions can contain levels of $\mathrm{SO}_{2}$ which are too low to be detected using current methods, and more sensitive techniques which utilise the available spectral information more effectively could make a useful contribution to current efforts.

As well as practical applications in hazard avoidance, BTD filters can provide useful information about the global distribution of important trace species using calibration methods to link the measured BTD to species abundance. For example, a BTD filter using IASI provided the first global measurements of tropospheric ammonia $\left(\mathrm{NH}_{3}\right)$, which has a large contribution from agriculture with negative impacts on the environment. Concentrations were determined taking the BTD between a target channel at $867.75 \mathrm{~cm}^{-1}$ in the $\mathrm{NH}_{3} \nu_{2}$ band and two background channels at 861.25 and $873.50 \mathrm{~cm}^{-1}$ using a limited number of full-retrievals to derive a scaling factor to convert the observed BTD's to estimated total column amounts (Clarisse et al., 2009).

The main difficulty in designing this type of BTD filter is the avoidance of false detections caused by variations in the non-target parameters such as interfering species, the temperature profile of the atmosphere, variations in surface temperature, and the presence of cloud and aerosol. This requires the careful selection of target and background channels which allow the unwanted contributions to the measured brightness temperature in the target channels to be eliminated. However, without some mathematical framework for balancing the various contributions it is only feasible to include a few channels, and the hyperspectral nature of the spectrometer is not exploited effectively resulting in much higher random error associated with instrument noise than could potentially be achieved. In addition, the threshold for a significant detection is not linked to the statistical significance of the observed BTD. Furthermore, there is no intrinsic way to estimate the abundance of the target species. This is instead done a posteriori by performing full-retrievals and extracting an empirical relationship between the retrieved values and the observed BTD which can then be applied over a wider area. Here we present a method which provides a framework for generating many channel filters with improved noise characteristics, which are not sensitively dependent on the particular channels used, and which intrinsically allow for an estimate of the abundance of the target gas to be derived in certain circumstances.

\section{Detection method}

In a given channel, the target signal is usually overwhelmed by changes in the spectral background due to variations in the unwanted parameters such as interfering species, atmospheric temperature, surface temperature, and cloudiness. In a standard retrieval, these parameters are retrieved together with the target so that their contribution can be accounted for. The aim of the method outlined in this paper is to separate the contribution of the target from the spectral background without having to retrieve any of these other parameters directly.

The scheme is essentially an optimally weighted one-step retrieval of the target column amount and a spectral offset. Adopting the notation in Rodgers (2000), we assume that the spectral measurements $\mathbf{y} \in \mathbb{R}^{m}$ can be represented by the forward model $F$ plus the total measurement error

$\boldsymbol{y}=F(\boldsymbol{x}, \boldsymbol{u})+\epsilon_{\mathrm{rnd}}+\epsilon_{\mathrm{sys}}$

where $\boldsymbol{x}=\left[x_{\mathrm{c}}, \text { off }\right]^{T}$ is the true state vector composed of the target species column amount $x_{\mathrm{c}}$ and a wavenumber independent brightness temperature offset off, $\boldsymbol{u}$ represents the best estimate of other parameters related to the instrument, atmosphere and surface, $\epsilon_{\text {rnd }}$ is the random measurement error determined by instrument noise, and $\epsilon_{\text {sys }}$ represents systematic measurement errors due to uncertainties in parameters $\boldsymbol{u}$. The spectral offset can be thought of as describing uncertainties in the parameters $\boldsymbol{u}$ which correspond approximately to broadband spectral contributions such as might arise from differences in the actual and modelled surface temperature, the presence of ash, and the cloudiness of the atmosphere. The forward model is linearised about a reference state with climatological levels of the target gas and our best knowledge of the parameters $\boldsymbol{u}$ where the detection is applied. When the forward model is nearly linear around the climatological atmospheric conditions, we can write

$\boldsymbol{y}-F\left(\boldsymbol{x}_{0}, \boldsymbol{u}\right)=\mathbf{K}\left(\boldsymbol{x}-\boldsymbol{x}_{0}\right)+\epsilon_{\mathrm{rnd}}+\epsilon_{\mathrm{sys}}$

where the linearisation point is taken to be $\boldsymbol{x}_{0}=\left[x_{\mathrm{c} 0}, 0\right]^{T}$ where $x_{\mathrm{c} 0}$ is a climatological column amount, and the $i$-th row of the jacobian $\mathbf{K} \in \mathbb{R}^{m \times 2}$ can be written as $\left[\frac{\partial y_{i}}{\partial x_{\mathrm{c}}}, 1\right]$ where $y_{i}$ are in terms of brightness temperature. The brightness temperature offset acts as a sink for some error terms which may be difficult to define in advance and correspond to broadband spectral offsets and should not be restricted to channels where the target species has a spectral contribution so that the baseline can be determined as effectively as possible. The optimal unconstrained least-squares estimate $\hat{\boldsymbol{x}}=\left[\hat{x}_{\mathrm{c}}, \hat{\mathrm{off}}^{T}\right.$ may then be computed as

$\hat{\boldsymbol{x}}=\boldsymbol{x}_{0}+\left(\mathbf{K}^{T} \mathbf{S}_{\epsilon}^{\mathrm{tot}-1} \mathbf{K}\right)^{-1} \mathbf{K}^{T} \mathbf{S}_{\epsilon}^{\mathrm{tot}-1}\left(\boldsymbol{y}-F\left(\boldsymbol{x}_{0}, \boldsymbol{u}\right)\right)$ 
where the matrix $\mathbf{S}_{\epsilon}^{\text {tot }} \in \mathbb{R}^{m \times m}$ is the covariance of the total error $\left(\epsilon^{\mathrm{rnd}}+\epsilon^{\mathrm{sys}}\right)$. The first row of the measurement contribution function

$\boldsymbol{G}=\left(\mathbf{K}^{T} \mathbf{S}_{\epsilon}^{\mathrm{tot}-1} \mathbf{K}\right)^{-1} \mathbf{K}^{T} \mathbf{S}_{\epsilon}^{\mathrm{tot}-1}$

is denoted $\mathbf{g}_{\mathrm{c}}^{T}$ and comprises the weights applied to a given spectrum to determine the presence of the target species. The total column amount given by $\hat{x}_{\mathrm{c}}$ is not intended to be an accurate retrieval of the target column amount but rather simply a metric for determining whether levels of the gas are enhanced with respect to the climatological background over the vertical levels where the instrument is sensitive. Other factors such as the conditions of thermal contrast with the surface where this is visible and how effectively the spectral background has been suppressed may also affect the measured column amount.

The use of the total measurement error covariance as a weights matrix for the least squares minimisation in Eq. (3) differs from the commonly adopted inversion process, which for reasons of computational efficiency uses only the random error covariance arising from instrument noise to weight measurements. Here, the inversion is weighted not only by instrument noise but also by systematic errors due to uncertainties in the forward model which arise from differences between the assumed forward model parameters and the physical radiative transfer process. These errors tend to be broadly spectrally correlated whereas the random error due to instrument noise is only locally correlated. For a single target parameter, if the inversion is weighted only by the random error due to measurement noise, then the solution is not optimal because the systematic errors due to uncertainties in the forward model are not accounted for. Sometimes these errors are small enough to be ignored, which is not the case here. Otherwise the uncertain parameters can be retrieved jointly with the target parameter increasing the size of the state vector to obtain an optimal solution. However, as explained in von Clarmann et al. (2001) an equivalent optimal solution can be obtained without increasing the size of the state vector, but instead by weighting measurements according to the total error, including the systematic errors. The diagonal elements of $\mathbf{S}_{\epsilon}^{\text {tot }}$ which contain the total random and systematic variance weight the spectral points against each other appropriately, assigning less weight to points with a large total error. The long-range correlations in the $\mathbf{S}_{\epsilon}^{\text {tot }}$ matrix, which arise from the systematic errors, then provide additional information about the expected behaviour of errors in one spectral point relative to the others. Physically, correlations in $\mathbf{S}_{\epsilon}^{\text {tot }}$ mean that a spectral point with very little target signal (and in this method even zero target signal) can still add significant information about the target species because it helps to fix the baseline in the target channels. By inverting the measurements in this way, enhancements in the target species can be identified and separated from the contribution of other unwanted parameters without having to retrieve the other parameters affecting the measurement. The results obtained by inverting the measurements in this way are similar to those which would be obtained by performing a joint retrieval of all parameters affecting the measurement.

Hence, for the detection method to work effectively, the total measurement error covariance used needs to be realistic. In this paper, this matrix is computed either by modelling errors using appropriate perturbations to the various physical parameters involved within the forward model, or by using an ensemble of spectra to compute the covariance of a large number of measured spectra with climatological levels of the target gas representative of the atmospheric conditions where the detection is applied.

\subsection{Modelling method}

The first approach, which does not rely on samples of measured spectra, involves the construction of $\mathbf{S}_{\epsilon}^{\text {tot }}$ considering simple perturbations to appropriate parameters within the forward model such as interfering species, atmospheric temperature, surface temperature, and cloudiness, which can then be combined with the random error determined from instrument noise to calculate an approximation of the total measurement error covariance

$\mathbf{S}_{\epsilon}^{\mathrm{tot}}=\mathbf{S}_{\epsilon}^{\mathrm{rnd}}+\mathbf{S}_{\epsilon}^{\mathrm{sys}} \approx \mathbf{S}_{\epsilon}^{\mathrm{rnd}}+\sum_{i=1}^{N} \mathbf{K}^{(i)} \mathbf{B}^{(i)} \mathbf{K}^{(i)^{T}}=\mathbf{S}_{\epsilon}^{\mathrm{mod}}$

where $\mathbf{S}_{\epsilon}^{\text {rnd }}$ is the random measurement error covariance determined by instrument noise and $\mathbf{S}_{\epsilon}^{\text {sys }}$ is the systematic error covariance due to forward model errors. The approximation of the total measurement error covariance calculated using the modelling method is denoted $\mathbf{S}_{\epsilon}^{\text {mod }}$ to distinguish it from another method introduced in Sect. 2.2 which uses an ensemble of measured spectra to estimate the total measurement error covariance.

The systematic component of measurement error is computed in general by considering the combination of $N$ error sources which are assumed to be independent. For each error source $i, \mathbf{S}_{\epsilon}^{\text {sys }}$ may be computed considering the $p \times p$ profile error covariance matrix $\mathbf{B}^{(i)}$ and the corresponding jacobian $\mathbf{K}^{(i)} \in \mathbb{R}^{m \times p}$. The profile error covariance matrix $\mathbf{B}^{(i)}$ could for example represent the climatological variability in a $p$ level profile of water vapour in which case $\mathbf{K}^{(i)} \in \mathbb{R}^{m \times p}$ is calculated around an appropriate value for the $p$ profile levels such that the jacobian contains the elements $\mathbf{K}_{j k}^{(i)}=\frac{\partial y_{j}}{\partial \mathrm{H}_{2} \mathrm{O}_{k}}$ where $1 \leq j \leq m$ and $1 \leq k \leq p$. This approach assumes linearity. If only the variance is available then the errors can be computed by perturbing the forward model directly to produce a series of error spectra for each error source $i$

$\Delta \boldsymbol{y}^{(i)}=F\left(\boldsymbol{x}_{0}, \boldsymbol{u}+\Delta \boldsymbol{u}^{(i)}\right)-F\left(\boldsymbol{x}_{0}, \boldsymbol{u}\right)$

where $\Delta \boldsymbol{u}^{(i)}$ is the estimated $1 \sigma$ variation in parameter $i$, and the corresponding error spectrum $\Delta \boldsymbol{y}^{(i)} \in \mathbb{R}^{m}$ can then 
be used to calculate an estimate of the systematic measurement error covariance and hence total measurement error covariance according to

$\mathbf{S}_{\epsilon}^{\mathrm{tot}}=\mathbf{S}_{\epsilon}^{\mathrm{rnd}}+\mathbf{S}_{\epsilon}^{\mathrm{sys}} \approx \mathbf{S}_{\epsilon}^{\mathrm{rnd}}+\sum_{i=1}^{N} \Delta \boldsymbol{y}^{(i)} \Delta \boldsymbol{y}^{(i) T}=\mathbf{S}_{\epsilon}^{\mathrm{mod}}$.

Ideally the modelled spectrum $F\left(\boldsymbol{x}_{0}, \boldsymbol{u}\right)$ in Eq. (3) should be equivalent to the observed spectrum for the climatological atmospheric conditions, including the target species concentration, interfering species concentration, atmospheric and surface temperature, conditions of cloudiness, presence of aerosols and so on. However, in practice this is difficult to achieve due to difficulties in defining and modelling the climatological atmospheric conditions. The modelled spectrum is therefore chosen for convenience to represent clearsky conditions, assuming that cloudiness, surface temperature, and other broadband features can be accounted for by retrieving the target jointly with a spectral offset.

All spectral modelling in this study was performed using the Reference Forward Model (RFM) using the HITRAN 2008 spectral database (Rothman et al., 2009). Details about the RFM can be found in the online manual (Dudhia, 2008).

\subsection{Ensemble method}

Using the ensemble approach, $\mathbf{S}_{\epsilon}^{\text {tot }}$ is estimated considering an appropriate ensemble of $N$ measured spectra to construct an estimate of the total measurement error covariance denoted $\mathbf{S}_{y}^{\text {obs }}$

$\mathbf{S}_{\epsilon}^{\mathrm{tot}} \approx \frac{1}{N-1} \sum_{j=1}^{N}\left(\boldsymbol{y}_{j}-\overline{\boldsymbol{y}}\right)\left(\boldsymbol{y}_{j}-\overline{\boldsymbol{y}}\right)^{T}=\mathbf{S}_{y}^{\mathrm{obs}}$

where $\bar{y}$ is the calculated mean spectrum for the ensemble

$\overline{\boldsymbol{y}}=\frac{1}{N} \sum_{j=1}^{N} \boldsymbol{y}_{j}$

However, this approach depends on the availability of an appropriate ensemble of spectra where there is some confidence that the signal from the target species is below the noise under normal circumstances. Volcanic $\mathrm{SO}_{2}$ in the free troposphere is a suitable candidate since the signal is well below the noise in the absence of a volcanic event, and the selection of an appropriate ensemble with background levels of the target species can be performed relatively easily. In the case where spectra which do contain enhancements are mistakenly included in the ensemble, this acts to reduce the sensitivity of the filter to the target species. Ammonia, however, is not suitable since strong sources are too widespread, making the selection of an appropriate ensemble difficult.

The mean spectrum $\bar{y}$ calculated in Eq. (8) should be used in place of $F\left(x_{0}, \boldsymbol{u}\right)$ in Eq. (3) when using the ensemble method as $\overline{\boldsymbol{y}}$ is a likely to be a better estimate of the climatological mean spectrum, and so ensures that the climatological value of the target species is retrieved when levels of the target species are not enhanced over those represented by the mean background spectrum $\overline{\boldsymbol{y}}$.

\subsection{Channel selection}

In most cases, the problem of which channels to use can be solved by a qualitative selection of a suitable block of channels. The method is then largely insensitive to the particular channels used within that block since the correlations in $\mathbf{S}_{\epsilon}^{\text {tot }}$ mean that all channels add information. The main factors to be considered are that the chosen region should not only include parts of the spectrum which have a relatively strong signal from the target species but also regions with little or no contribution from the target to maximise the available contrast with the spectral background. Spectral regions which cannot be modelled adequately should be avoided where possible.

In order to confirm that none of the channels in the chosen region have a detrimental impact on the detection, the information content about the target species may be checked as more channels are added. The first step involves finding the top two channels in the chosen region with the lowest total retrieval error variance $\sigma_{\mathrm{c}}^{2}$ for the target component by searching through all permutations of channel pairs propagating the total measurement error through the retrieval according to

$\mathbf{S}_{x}^{\mathrm{tot}}=\left(\mathbf{K}^{T} \mathbf{S}_{\epsilon}^{\mathrm{tot}-1} \mathbf{K}\right)^{-1}$

where $\mathbf{S}_{x}^{\text {tot }}$ is the total retrieval error covariance. The pair of channels with the lowest target total retrieval error variance $\sigma_{\mathrm{c}}^{2}$ is then assessed in combination with each remaining channel. The best combination of those channels may then be assessed by computing the information content $H$ in bits obtained about the target parameter by including the additional channel

$H=\frac{1}{2} \log _{2}\left(\frac{\left(\sigma_{\mathrm{c}}^{2}\right)_{i+1}}{\left(\sigma_{\mathrm{c}}^{2}\right)_{i}}\right)$

where $\left(\sigma_{\mathrm{c}}^{2}\right)_{i}$ is the total retrieval error variance for the target species computed for $i$ channels and $\left(\sigma_{\mathrm{c}}^{2}\right)_{i+1}$ is the total retrieval error variance for the target species computed for $i+1$ channels. Channels which contribute negligible information about the target parameter can then be excluded as a matter of efficiency if required.

\subsection{Detection threshold}

Once a suitable set of channels have been defined, the apparent target column amount given in Eq. (3) may be compared against a threshold which indicates a positive detection with a certain confidence. An approximate estimate of the level of 
significance of a particular detection can be gauged by comparison of the enhancement, $\hat{x}_{\mathrm{c}}-x_{\mathrm{c} 0}$, against the square root of the sum of the total retrieval error variance for the target gas column and the climatological variance of the target species. In the cases examined in this paper, however, the target species variability is negligible compared to the sensitivity of the instrument under climatological conditions, and so it is only necessary to compare the enhancement against the total retrieval error variance for the target column $\sigma_{\mathrm{c}}{ }^{2}$. From Eqs. (3) and (4), it can be seen that the difference between the detected column and the climatological column corresponds to $\boldsymbol{g}_{\mathrm{c}}^{T} \boldsymbol{y}-x_{\mathrm{c} 0}$. Hence, we can calculate the significance of a particular detection in terms of how far away a particular observation is from the climatology in terms of number of total retrieval error standard deviations

$Z_{\mathrm{obs}}=\frac{\boldsymbol{g}_{\mathrm{c}}^{T} \boldsymbol{y}-x_{\mathrm{c} 0}}{\sigma_{\mathrm{c}}}$.

Depending on the application, a suitable $Z$-number can be chosen so that there is a positive detection when the following condition is satisfied

$\hat{x}_{\mathrm{c}}>x_{\mathrm{c} 0}+Z_{\text {thresh }} \sigma_{\mathrm{c}}$.

\section{Application of the filters}

Trace species detection filters generated using the methods described above were applied to IASI spectra for two case studies: (a) the emission of $\mathrm{SO}_{2}$ from the eruption of the Kasatochi volcano in Alaska in August 2008, and (b) the emission of $\mathrm{NH}_{3}$ from agriculture over Southern Asia in May 2008. Before application of the filters, an appropriate viewing angle correction was applied to spectra obtained in the sideways views which have a greater path length through the atmosphere. The measured spectra were scaled by an airmass factor $\cos (\phi)$ where $\phi$ is the ground angle defined as the angle between the satellite and the local vertical at the ground at the location of the observation. The ground angle may be calculated from trigonometric considerations assuming a spherical Earth as

$\phi=\sin ^{-1}\left(\frac{\sin (\theta)\left(R_{\mathrm{E}}+h\right)}{R_{\mathrm{E}}}\right)$.

\subsection{Case study 1: the detection of volcanic emissions}

The detection method was tested for $\mathrm{SO}_{2}$ from the eruption of the Kasatochi Volcano in the Aleucian Islands, Alaska, in early August 2008. Ordinarily, $\mathrm{SO}_{2}$ is concentrated in the boundary layer with total column amounts of less than $0.1 \mathrm{DU}$. Sources of $\mathrm{SO}_{2}$ contributing to background levels in the lower troposphere include the slow degassing from volcanoes, burning of fossil fuels and biomass, and tin smelting. There are two emission features in the thermal infra-red: the symmetric stretch $v_{1}$ band centred on $1152 \mathrm{~cm}^{-1}$ and the
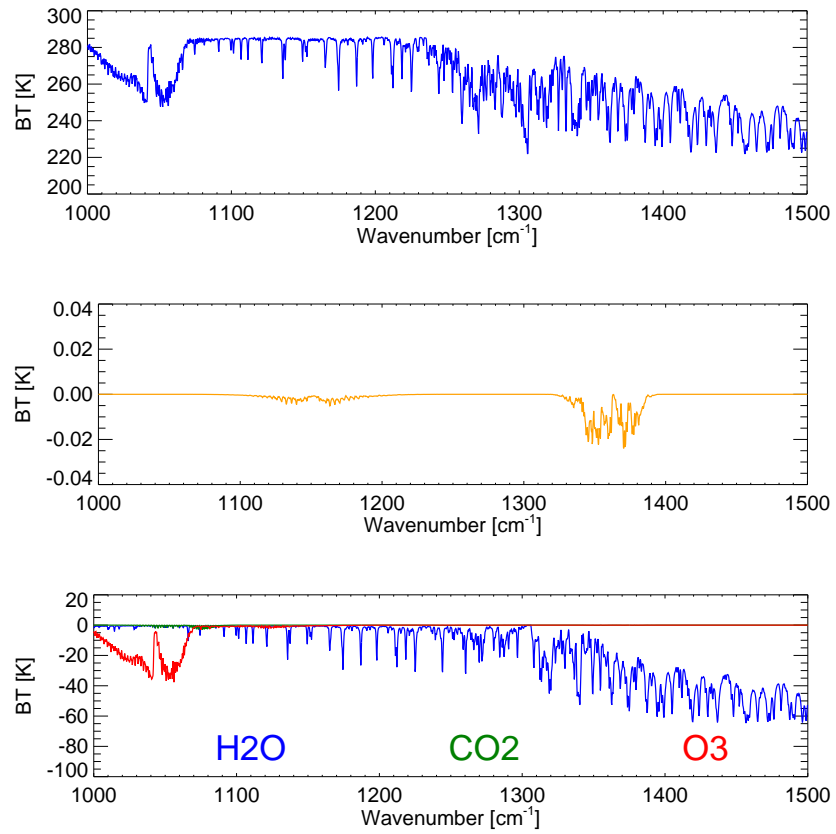

Fig. 1. Simulated spectra in the region of the $\mathrm{SO}_{2} v_{1}$ and $v_{3}$ bands. Top panel shows a simulated spectrum for a standard atmosphere. Middle panel shows contribution of climatological background levels of $\mathrm{SO}_{2}$. Bottom panel shows contribution of main interfering species (water vapour, $\mathrm{CO}_{2}$ and ozone).

antisymmetric stretch $\nu_{3}$ band centred on $1362 \mathrm{~cm}^{-1}$. As shown in Fig. 1, the spectral contribution of climatological concentrations of $\mathrm{SO}_{2}$ is up to $0.005 \mathrm{~K}$ in the $v_{1}$ band and $0.025 \mathrm{~K}$ in the $\nu_{3}$ band. The nominal NE $\Delta T$ at $280 \mathrm{~K}$ between $1000-1200 \mathrm{~cm}^{-1}$ ranges between 0.950 and $0.165 \mathrm{~K}$ and the NE $\Delta T$ at $280 \mathrm{~K}$ between $1300-1410 \mathrm{~cm}^{-1}$ ranges between 0.980 and $0.105 \mathrm{~K}$ (Cayla et al., 1995). Therefore, the signal from typical background levels of $\mathrm{SO}_{2}$ are well below the noise level of the instrument. Absorption in the $v_{1}$ band is not as strong as absorption in the $v_{3}$ band. However, the atmosphere is more transparent in the region of the $v_{1}$ band. The $v_{3}$ feature, on the other hand, is obscured by strong water vapour absorption, and so there is negligible sensitivity to $\mathrm{SO}_{2}$ in the lower troposphere in this spectral region.

The eruption of Kasatochi beginning on 7 August 2008 caused significant disruption to air-traffic due to the volcanic ash emitted (Guffanti et al., 2008). On 8 August, a plume of ash and $\mathrm{SO}_{2}$ was observed at an estimated altitude of $12.5 \pm 4 \mathrm{~km}$, with total column amounts of $\mathrm{SO}_{2}$ estimated at $311 \mathrm{DU}$ (Karagulian et al., 2010). Two days later the plume had spread southward into the Pacific and OMI/Aura measured maximum column amounts of 105 DU (OMI/Aura [Internet], 2010). Figure 2 shows examples of spectra acquired inside and outside of the volcanic plume on this date. The IASI instrument is easily sensitive enough to detect the very large signal due to $\mathrm{SO}_{2}$ within the plume. However, 


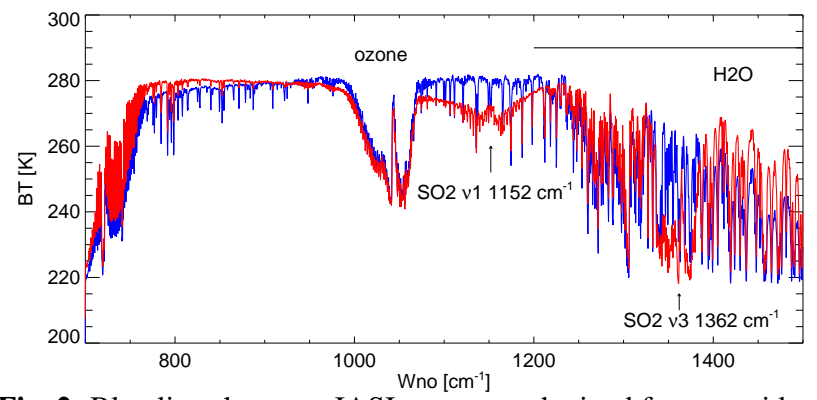

Fig. 2. Blue line shows an IASI spectrum obtained from outside of the Kasatochi volcanic plume on 10 August over ocean and the red line shows an IASI spectrum obtained inside the plume.

the challenge in designing detection filters is to make them as insensitive as possible to unwanted parameters such as clouds and water vapour, which have a large spectral contribution and are highly variable. Therefore, for each filter we also examine the distribution of values in a region of atmosphere outside the plume to test how well these other parameters have been suppressed. Ideally, the climatological total column amount of $\mathrm{SO}_{2}$ should be recovered $(=0.076 \mathrm{DU})$ outside the plume.

The results using the existing four channel filter are shown in Fig. 3 for the evening of 10 August 2008. The variation in the background values in a selected region outside of the plume normalised with respect to the maximum BTD observed inside the plume $(48 \mathrm{~K})$ are also shown. The existing filter performs very well and the variation in the spectral background is generally less than $1 \%$ of the maximum value in the plume. In the following sections we examine whether it is possible to improve the sensitivity of the detection still further.

\subsection{1 $v_{1}$ band filter}

A filter was constructed using all channels between 1000 $1200 \mathrm{~cm}^{-1}$ in the vicinity of the $\mathrm{SO}_{2} v_{1}$ band. Checking the information content of the filter using the approach described in Sect. 2.3 indicates that all channels in this region contribute information. The entire measurement block was therefore used to generate the filter. The region around the $\mathrm{SO}_{2} v_{1}$ band is optically thin provided that the atmosphere is clear of ash and cloud, with some sensitivity to the lower troposphere. The region avoids an ice signature present mainly between $800-1000 \mathrm{~cm}^{-1}$. However, the region overlaps strong reflections from the quartz Reststrahlung band between $1100-1250 \mathrm{~cm}^{-1}$ (E. Pavelin, Met. Office, personal communication, 2010) which have much sharper spectral dependencies, which cannot at present be modelled using the RFM, and mean the filter should not be used over some land surfaces in arid regions. Two approaches are considered for the construction of the $\mathrm{SO}_{2}$ filter using the $v_{1}$ band:

- Method 1: The $\mathbf{S}_{\epsilon}^{\text {tot }}$ matrix in Eq. (3) is constructed using the modelling approach $\left(\mathbf{S}_{\epsilon}^{\text {tot }} \approx \mathbf{S}_{\epsilon}^{\text {mod }}\right)$ as in Eqs. (6)
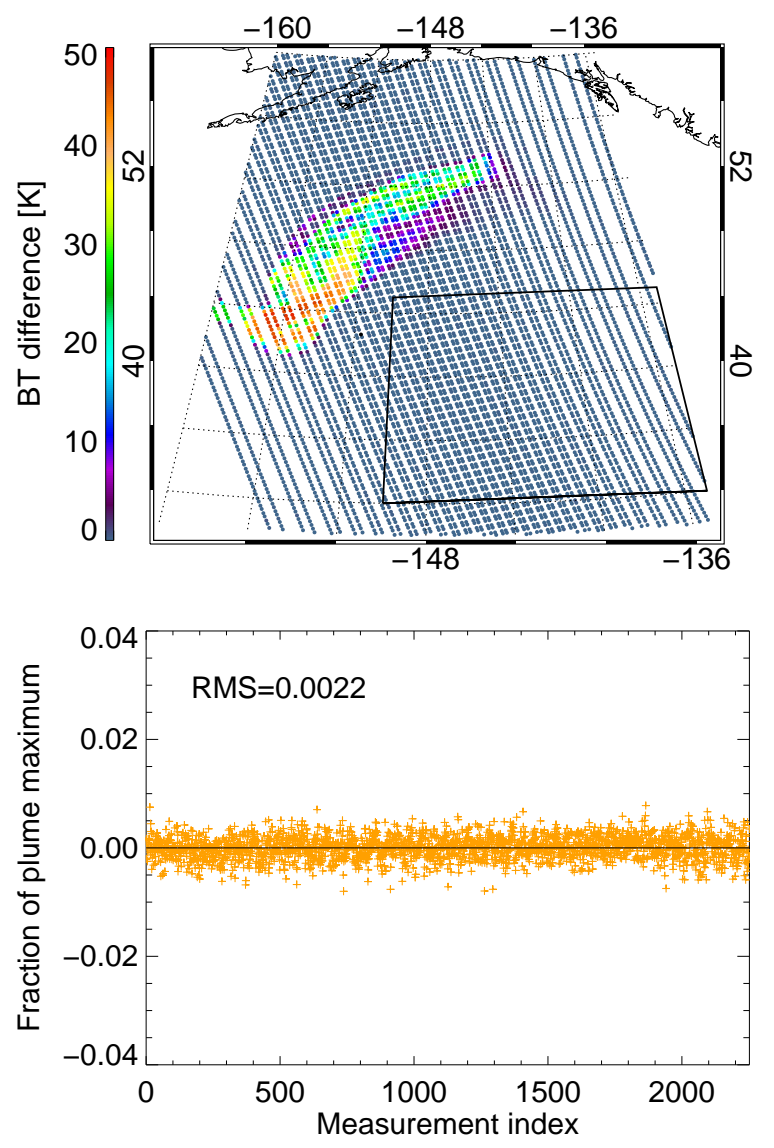

Fig. 3. Detection of $\mathrm{SO}_{2}$ plume for the Kasatochi eruption for the morning overpass on 10 August 2008 using the four channel BTD by Clarisse et al. (2008) in terms of background channels minus $\mathrm{SO}_{2}$ sensitive channels. The variation in the spectral background within the box is shown in the bottom panel in terms of the fractional variation normalised with respect to the maximum BT value observed inside the plume $(48 \mathrm{~K})$. Observed RMS of background $=0.0022$.

and (7) considering simple perturbations to forward model parameters. Climatological $1 \sigma$ column perturbations are applied to temperature and interfering species with variability above the level of instrument noise (mainly ozone and $\mathrm{H}_{2} \mathrm{O}$ ) using the profile uncertainties defined for the mid-latitude atmosphere in the IG2 climatological database (Remedios et al., 2007), which is also used as the linearisation point $\left[\boldsymbol{x}_{0}, \boldsymbol{u}\right]$. A $20 \mathrm{~K}$ perturbation is applied to surface temperature, and variability due to cloudiness is modelled using 15 independent homogenous optically thick cloud layers at $1 \mathrm{~km}$ intervals from $1-15 \mathrm{~km}$. Each error source is assumed to be independent of the others.

- Method 2: The $\mathbf{S}_{\epsilon}^{\text {tot }}$ matrix is constructed using the ensemble approach ( $\left.\mathbf{S}_{\epsilon}^{\text {tot }} \approx \mathbf{S}_{y}^{\text {obs }}\right)$ as in Eqs. (8) and (9) using an ensemble of 2253 measured spectra in a region 
without $\mathrm{SO}_{2}$ enhancements in the box bounded by the [lat, lon] coordinates [32, 45] and $[-150,-135]$ on the 10 August 2008 to construct an estimate of the total measurement error covariance matrix for the background atmosphere in the vicinity of the volcanic plume.

Figure 4 shows the $\mathrm{SO}_{2}$ detected using the $\nu_{1}$ band filter where $\mathbf{S}_{\epsilon}^{\text {tot }}$ is constructed using Method (1) using the modelling approach. The plume of $\mathrm{SO}_{2}$ is clearly visible in the Pacific Ocean. The shape of the plume appears broadly similar to that observed using the four-channel filter in Fig. 3 (which uses the $v_{3}$ band rather than the $v_{1}$ band), although the extent of plume appears to be somewhat more restricted in this case. Absorption in the $v_{1}$ band is not as strong as for the $v_{3}$ band feature. However, the atmosphere is more transparent in the region of the $v_{1}$ band, except in the presence of ash, whereas in the region of the $\nu_{3}$ band feature there is very strong overlapping absorption due to water vapour. It is likely that the apparent reduced geographical extent of the plume as observed using the $v_{1}$ band filter is due to reduced sensitivity of the $v_{1}$ band compared to the $v_{3}$ band at higher altitudes for $\mathrm{SO}_{2}$ observed above the dense volcanic ash layer that was present in this plume. The maximum value observed inside the plume against which the background values were normalised was $326 \mathrm{DU}$. The normalised variation in the background within the box shown in Fig. 4 does not show any obvious structure and the values appear to be normally distributed which indicates that the variations in the spectral background due to parameters such as ozone and cloudiness have been effectively suppressed. There is a very slight negative offset in the distribution of the values which in the normalised fractional units is reported as -0.0019 rather than the expected $0.0023(=0.076 \mathrm{DU})$ if the expected climatological amount of $\mathrm{SO}_{2}$ were to be retrieved. The reported $1 \sigma$ variation for this filter is $0.0022(=0.734 \mathrm{DU})$ which is slightly less than the observed RMS of the background field of 0.0055 .

The detection of $\mathrm{SO}_{2}$ using the $v_{1}$ band where $\mathbf{S}_{\epsilon}^{\text {tot }}$ is constructed using the ensemble approach explained in Method (2) is shown in Fig. 5. It is assumed that the weights calculated using this ensemble are representative of a wider area including the plume and surrounding background observations. The maximum value observed inside the plume in this case was 328 DU. Since the background values shown in the figure were used as the ensemble to generate the filter, this represents the ideal case of what can be achieved in terms of suppression of the spectral background. The reported $1 \sigma$ sensitivity of $0.0024(0.812 \mathrm{DU})$ of the filter matches the observed RMS of the background which was computed as 0.0024 , and the mean value of the background observations yields the climatological value of $\mathrm{SO}_{2}$, as expected.
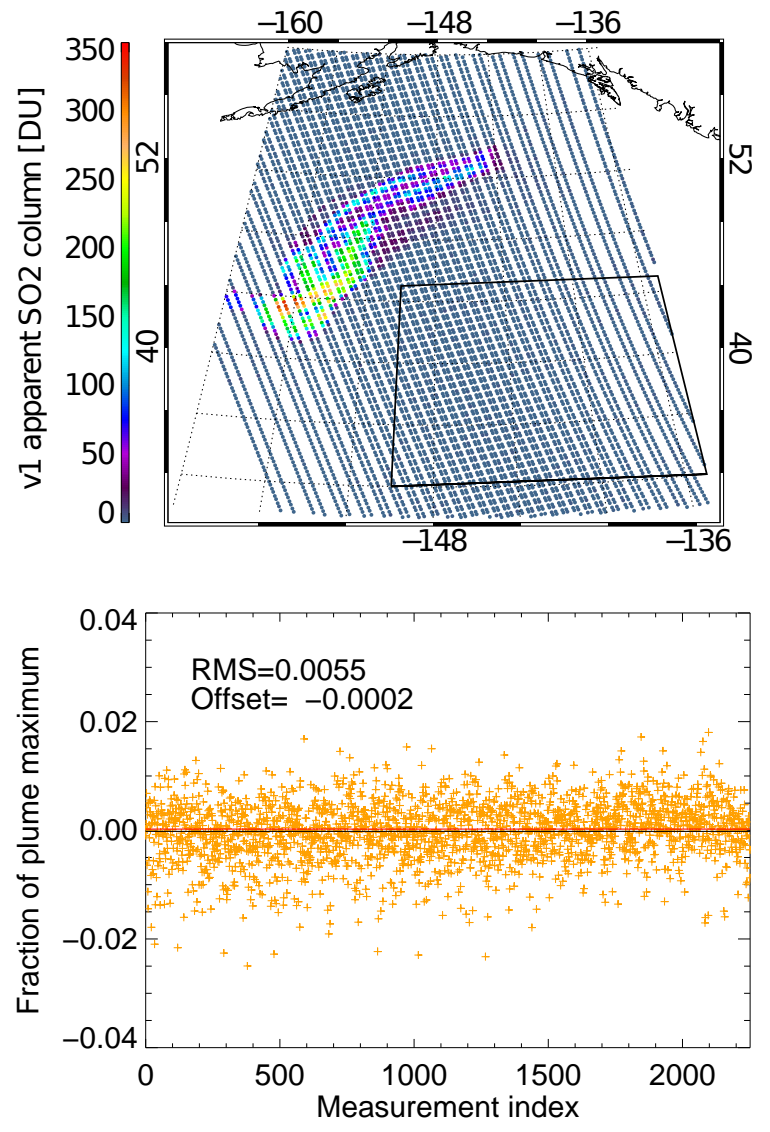

Fig. 4. The plume of $\mathrm{SO}_{2}$ for the morning overpass on $10 \mathrm{Au}-$ gust 2008 using the $v_{1}$ band filter for Method (1) as described in the text using the modelling approach to compute $\mathbf{S}_{\epsilon}^{\text {tot }}$. Fractional variation in the background normalised according to the maximum value within the plume (326 DU) shown in the bottom panel for IFOV's inside box. Reported $1 \sigma$ sensitivity $\left(\sigma_{\mathrm{c}}\right)$ for this filter of 0.0022 $(=0.734 \mathrm{DU})$. Observed RMS of background $=0.0055$. Expected climatological value shown as red line. Mean value of background value shown as black line

\subsection{2 $v_{3}$ band}

The $\nu_{3}$ band filter uses all channels between 1300-1410 $\mathrm{cm}^{-1}$. The $v_{3}$ band is generally more sensitive to $\mathrm{SO}_{2}$ than the $v_{1}$ band, except in the lower troposphere, which is mostly obscured by interference from optically thick water vapour lines. Since there are no sharp emission features not modelled by the RFM and the surface cannot be seen, there are no restrictions on surface type. However, as can be seen in Fig. 2, the $v_{3}$ band filter sits on the edge of the water vapour band, and is therefore sensitive to variability throughout the troposphere. For this reason, $\mathbf{S}_{\epsilon}^{\text {tot }}$ is more difficult to parameterise using the modelling approach. The main problem is variability due to water vapour itself since this accounts for over two orders of magnitude more variance than the other parameters. Three approaches are therefore considered which mainly differ in their treatment of water vapour: 

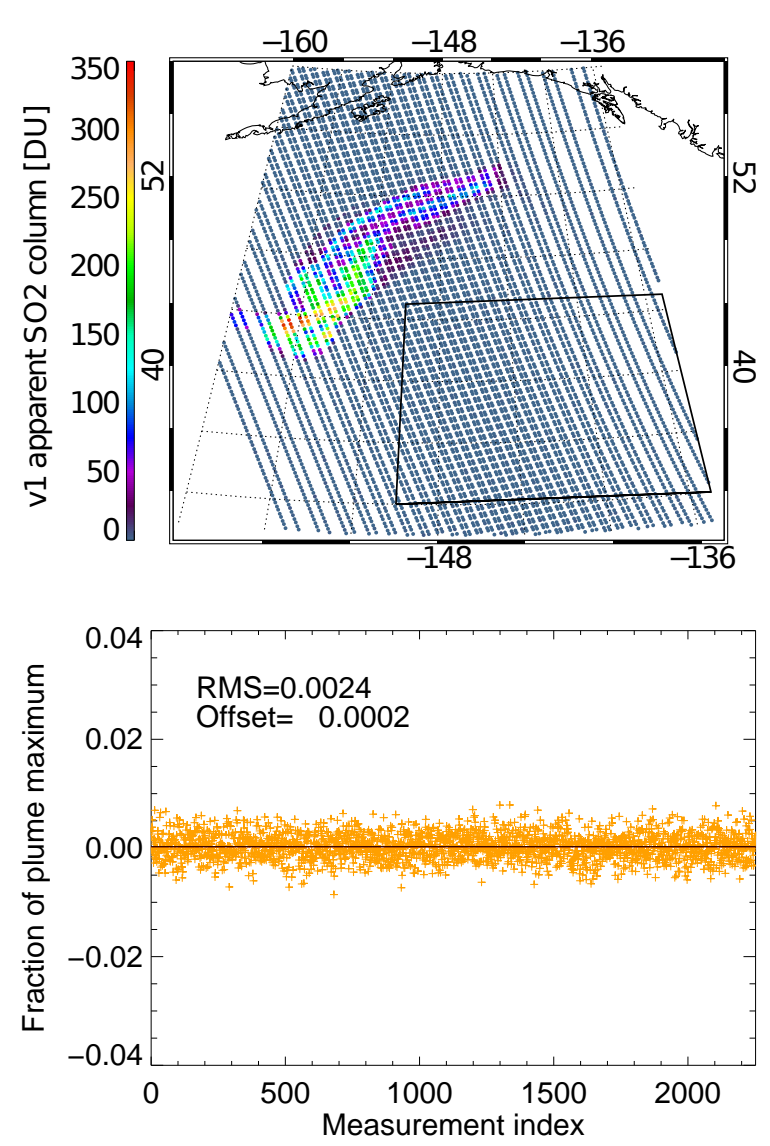

Fig. 5. The plume of $\mathrm{SO}_{2}$ for the morning overpass on $10 \mathrm{Au}$ gust 2008 using the $v_{1}$ band filter for Method (2) as described in the text using the ensemble approach to compute $\mathbf{S}_{\epsilon}^{\text {tot }}$. Fractional variation in the background normalised according to the maximum value within the plume (328 DU) shown in the bottom panel for IFOV's inside box. Reported $1 \sigma$ sensitivity $\left(\sigma_{\mathrm{c}}\right)$ for this filter of 0.0024 $(=0.812 \mathrm{DU})$. Observed RMS of background $=0.0024$. Expected climatological value shown as red line. Mean value of background value shown as black line

- Method 1: $\mathbf{S}_{\epsilon}^{\text {tot }}$ is constructed according to Eqs. (6) and (7) considering climatological $1 \sigma$ column perturbations to the temperature and water vapour profiles within the forward model as defined in the mid-latitude atmosphere in the IG2 climatological database (Remedios et al., 2007), which is also used as the linearisation point. A $20 \mathrm{~K}$ perturbation is applied to surface temperature, and perturbations representing 15 independent homogenous optically thick cloud layers at $1 \mathrm{~km}$ intervals from $1-15 \mathrm{~km}$ are included.

- Method 2: As in Method 1 but now $\mathbf{S}_{\epsilon}^{\text {tot }}$ is constructed as in Eq. (5) considering a $\mathbf{B}$ matrix for water vapour on 27 levels corresponding to the standard covariance used in the Met Office NWP SAF 1D-Var scheme. Temperature is modelled similarly to water vapour but considering a $\mathbf{B}$ matrix on 44 levels.
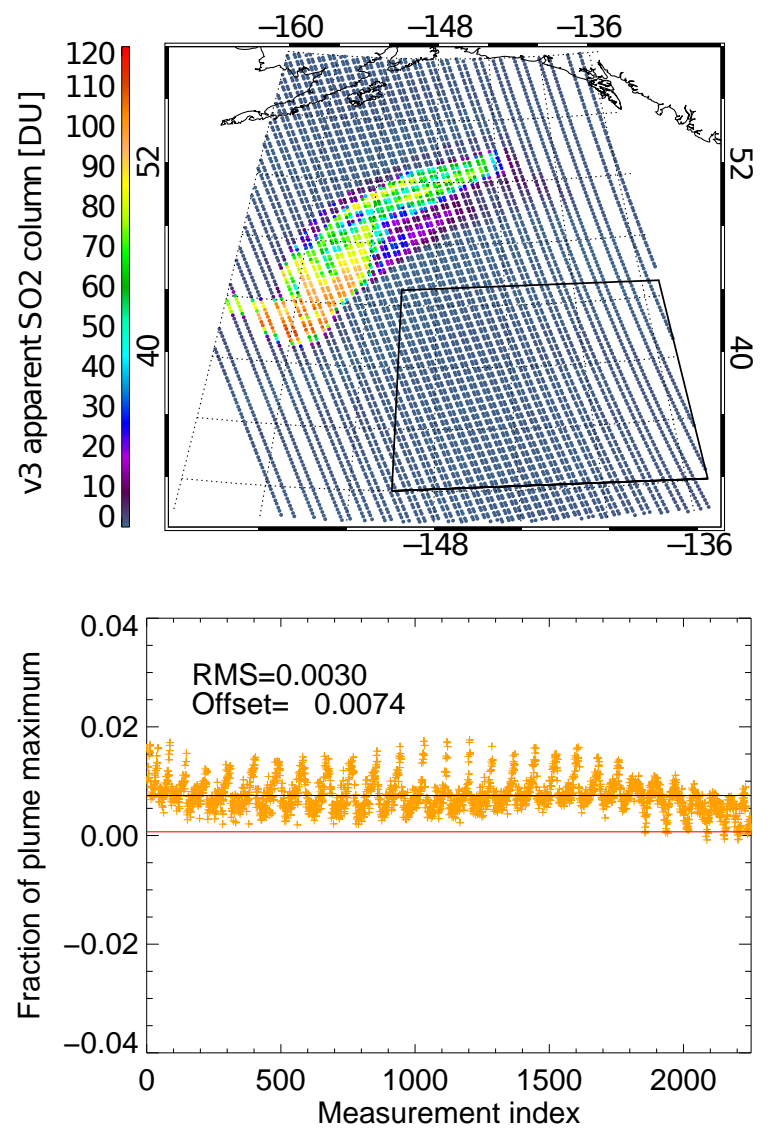

Fig. 6. The plume of $\mathrm{SO}_{2}$ for the morning overpass on $10 \mathrm{Au}$ gust 2008 using the $v_{3}$ band filter for Method (1) as described in the text using the modelling approach to compute $\mathbf{S}_{\epsilon}^{\text {tot }}$. Fractional variation in the background normalised according to the maximum value within the plume (109 DU) shown in the bottom panel for IFOV's inside box. Reported $1 \sigma$ sensitivity $\left(\sigma_{\mathrm{c}}\right)$ for this filter of 0.0013 $(=0.122 \mathrm{DU})$. Observed RMS of background $=0.0030$. Expected climatological value shown as red line. Mean value of background value shown as black line.

- Method 3: The $\mathbf{S}_{\epsilon}^{\text {tot }}$ matrix is constructed using an ensemble of 2253 measured spectra according to Eqs. (8) and (9) in a region without $\mathrm{SO}_{2}$ enhancements in the box bounded by the [lat, lon] coordinates [32, 45] and $[-150,-135]$ on 10 August 2008 to construct an estimate of the total measurement error covariance matrix for the background atmosphere in the vicinity of the eruption.

Figure 6 shows the results obtained for Method (1) described above whereby water vapour variability is modelled considering a $1 \sigma$ column perturbation. The maximum value observed inside the plume used to normalise the background values was $109 \mathrm{DU}$. Although the variation in the background without $\mathrm{SO}_{2}$ enhancements is mostly less than $1 \%$, there are some clear systematic drifts in the retrieved background. Firstly, the mean value of the background is higher 

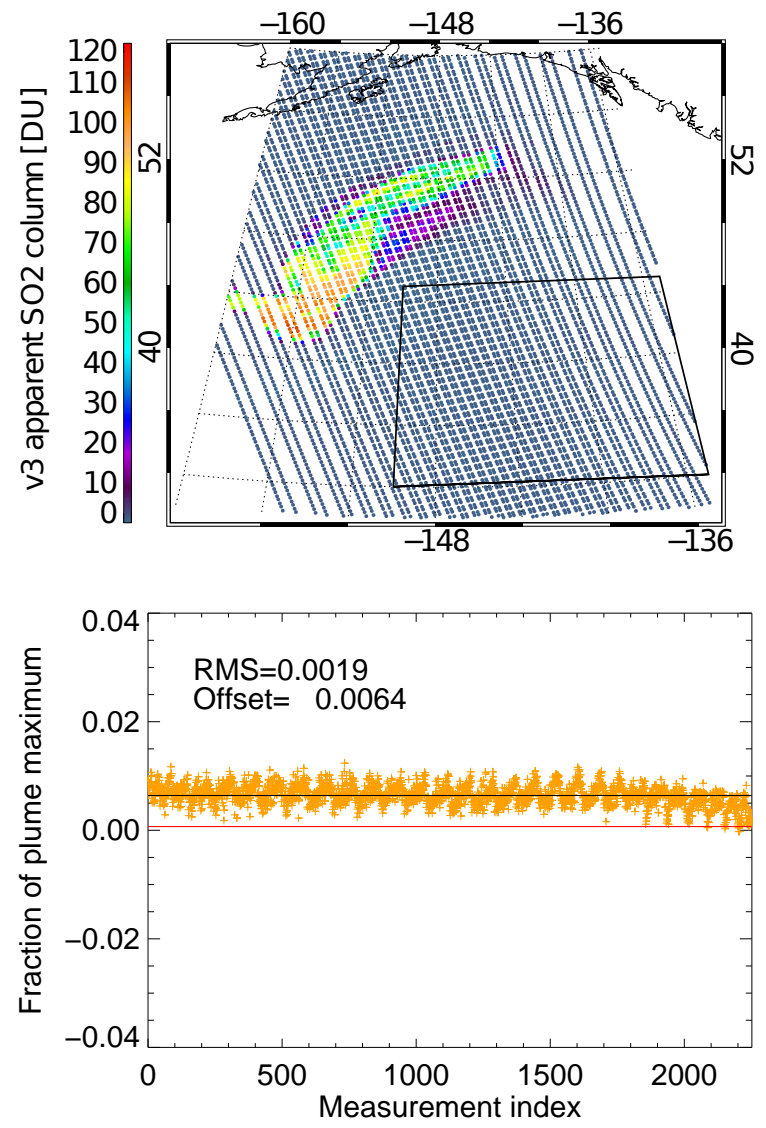

Fig. 7. The plume of $\mathrm{SO}_{2}$ for the morning overpass on $10 \mathrm{Au}$ gust 2008 using the $\nu_{3}$ band filter for Method (2) as described in the text using the modelling approach to compute $\mathbf{S}_{\epsilon}^{\text {tot }}$. Fractional variation in the background normalised according to the maximum value within the plume (108 DU) shown in the bottom panel for IFOV's inside box. Reported $1 \sigma$ sensitivity $\left(\sigma_{\mathrm{c}}\right)$ for this filter of 0.0012 $(=0.126 \mathrm{DU})$. Observed RMS of background $=0.0019$. Expected climatological value shown as red line. Mean value of background value shown as black line.

than the expected climatological background with a positive offset of 0.0064 rather than the expected 0.0022 in fractional units. In addition the values vary periodically. These variations are associated with the sideways viewing function of the instrument. In the case of sensitivity to different levels in the troposphere, a simple viewing angle correction applied to the measured spectra cannot fully correct for changes in observed radiance with satellite zenith angle. There are also other drifts in the background values not associated with the sideways viewing function of the instrument. Upon inspection it was found that these drifts in the background correspond to gaps between clouds, where the measurements are sensitive to water vapour throughout the troposphere. It is likely that in this case a column perturbation of water vapour is not a good enough representation of the variability. Instead, therefore, in Method (2) a perturbation is applied to
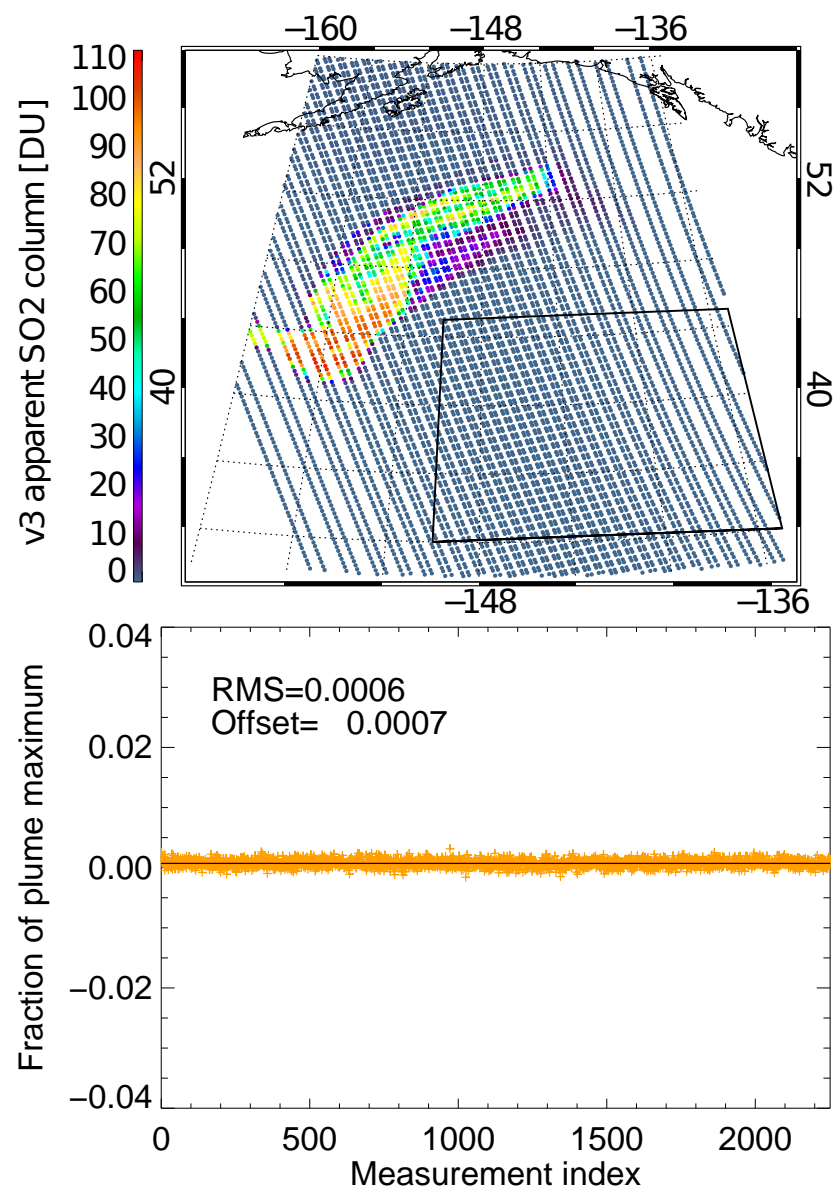

Fig. 8. The plume of $\mathrm{SO}_{2}$ for the morning overpass on $10 \mathrm{Au}$ gust 2008 using the $v_{3}$ band filter for Method (3) as described in the text using the ensemble approach to compute $\mathbf{S}_{\epsilon}^{\text {tot }}$. Fractional variation in the background normalised according to the maximum value within the plume (107 DU) shown in the bottom panel for IFOV's inside box. Reported $1 \sigma$ sensitivity $\left(\sigma_{\mathrm{c}}\right)$ for this filter of 0.0006 $(=0.067 \mathrm{DU})$. Observed RMS of background $=0.0006$. Expected climatological value shown as red line. Mean value of background value shown as black line

water vapour considering a $\mathbf{B}$ matrix with more realistic correlations between vertical levels. In this case, the systematic drifts in the background appear to be slightly improved over Method (1) as can be seen from Fig. 7.

However, the best results are obtained using the ensemble method described in Method (3) as shown in Fig. 8. The maximum value observed inside the plume in this case was 107 DU. Since both nadir viewing and sideways viewing measurements are used in the ensemble, the periodicity in the background values associated with the sideways scanning of the instrument is now also suppressed, and other drifts associated with the inadequate representation of the vertical variability in the troposphere in the modelling approach have now also disappeared. Here, the distribution of the background values is normally distributed around a 
typical climatological value of the $\mathrm{SO}_{2}$ column. Using this filter, the variations in the background values are mostly below $0.1 \%$ of the maximum apparent column amount in the plume. This is around an order of magnitude more sensitive than both the $v_{1}$ band and four channel filters.

For the detection of volcanic $\mathrm{SO}_{2}$ using the $v_{3}$ band, the ensemble method produces more sensitive detections than the modelling method since the total measurement error covariance matrix is more realistic and the spectral background is suppressed more effectively. In the cases examined above, the filters were generated from spectra in a region with no enhancements in $\mathrm{SO}_{2}$, and these filters were then applied to the entire scene to highlight a region of volcanic $\mathrm{SO}_{2}$ at a distant location. However, the filter is not limited to use in a single scene. As long as the ensemble contains enough members to capture the statistical properties of the variability of water vapour, cloudiness, and temperature effects, the filter can be reused and applied in another scene. It may even be possible to construct a filter which is applicable globally through consideration of a very large ensemble encompassing the full range of atmospheric conditions, although the sensitivity of the filter would be reduced due to the greater variability encompassed by the ensemble. The best approach may be to construct several filters for use during different times of year in appropriate geographical regions. This should be possible since the statistical properties of the background field change over large distances for example polar, mid-latitude and equatorial regimes, and on the seasonal time scale, and although some care would be needed to ensure an appropriate ensemble is selected for each situation, there is no reason why one $\mathbf{S}_{y}^{\text {obs }}$ cannot be used over a fairly large geographical region for an extended period of time as long as the statistical properties of the variability of the background atmosphere are nearly the same. For a global $\mathrm{SO}_{2}$ detection, several different ensembles would probably be necessary e.g., deserts would require a separate filter from equatorial scenes, and seasonal changes would need to be accounted for, essentially constructing a climatology of water vapour, cloudiness and temperature conditions stored as $\mathbf{S}_{y}^{\mathrm{obs}}$ matrices.

\subsection{Case study 2: emissions from agriculture}

The first global distributions of tropospheric ammonia were derived using the $\nu_{2}$ vibrational band taking the BTD at $867.75 \mathrm{~cm}^{-1}$ and two background channels at 861.25 and $873.50 \mathrm{~cm}^{-1}$ (Clarisse et al., 2009). The gas is present mainly in the boundary layer with very low concentrations higher in the troposphere. On the time-scale of days, it is converted to ammonium sulphate and ammonium nitrate which then play a role in the long-range transport of acidic pollutants (Sutton et al., 2009). We focus on a region of Southern Asia identified by Clarisse et al. (2009) as an area containing strong sources of ammonia from agriculture and compare the results obtained using the three channel filter against results obtained using the new filter.
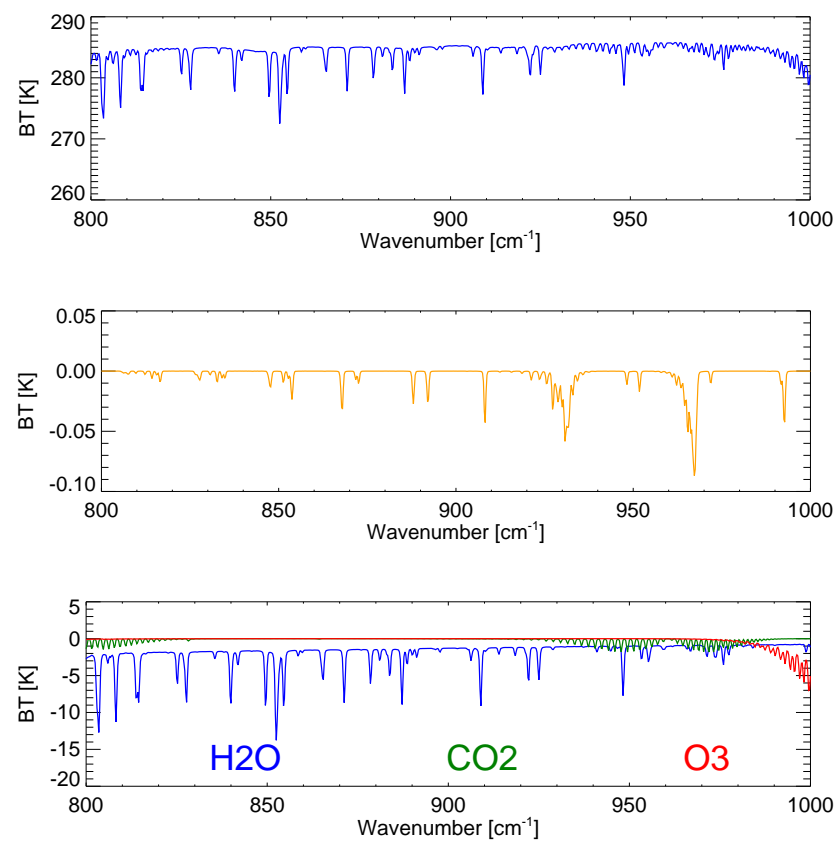

Fig. 9. Simulated spectra in the region of the ammonia $v_{2}$ band. Top panel shows a simulated spectrum for a standard atmosphere. Middle panel shows contribution of background levels of ammonia. Bottom panel shows contribution of main interfering species (water vapour, $\mathrm{CO}_{2}$ and ozone).

The new detection filter for ammonia was created using the $\nu_{2}$ band centred on $967 \mathrm{~cm}^{-1}$ including all channels between 800 and $1000 \mathrm{~cm}^{-1}$ using the modelling method to estimate $\mathbf{S}_{\epsilon}^{\text {tot }}$. Here we use the modelling method as the ensemble method is more difficult to apply in this case due to the more diffuse distribution of detectable ammonia in the atmosphere. As can be seen from Fig. 9, this spectral region is optically thin in the absence of cloud. The nominal NE $\Delta T$ at $280 \mathrm{~K}$ between $800-900 \mathrm{~cm}^{-1}$ is between $0.145-0.150 \mathrm{~K}$ (Cayla et al., 1995), and so background concentrations of ammonia such as are observed in non-polluted regions or over ocean are expected to be well below the noise level of the instrument.

To construct $\mathbf{S}_{\epsilon}^{\text {mod }}$, variability due to interfering species was modelled considering $1 \sigma$ total column perturbations to water vapour, ozone, and carbon dioxide as defined in the mid-latitude IG2 climatology which was also used as the linearisation point. Variability due to atmospheric temperature was modelled considering a $1 \sigma$ column perturbation. Surface temperature and emissivity variability (assuming no spectral dependence) was modelled considering a $20 \mathrm{~K}$ perturbation and zero thermal contrast was assumed with the first atmospheric layer. Cloudiness was modelled considering 15 independent optically thick cloud layers in the troposphere between 1 and $15 \mathrm{~km}$.

The ammonia filters were applied across a region of Asia in an area with persistent, high concentrations of 


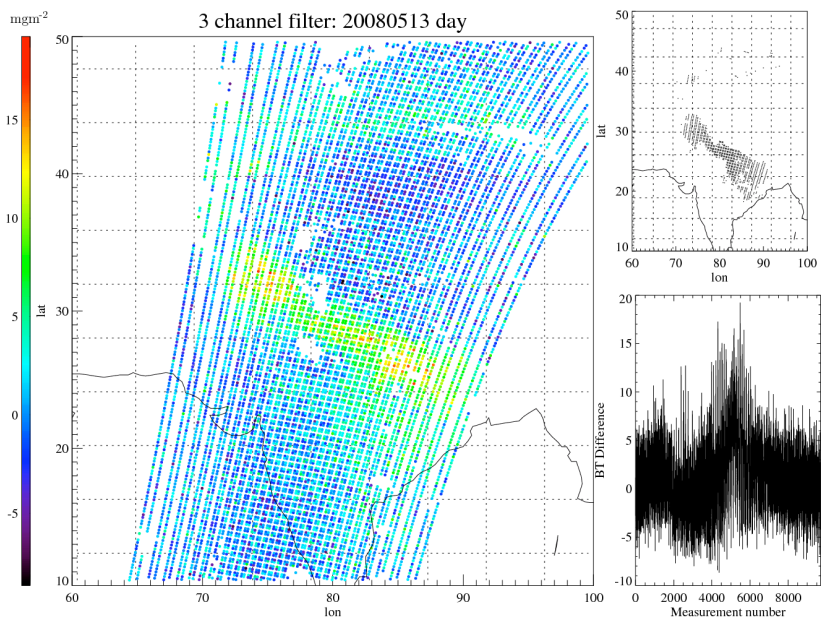

Fig. 10. Ammonia detected across Asia for the morning overpass on 13 May 2008 using the existing three channel filter with estimated total column amounts given in $\mathrm{mg} \mathrm{m}^{-2}$. A map of positive detections in the $99 \%$ confidence interval is shown in the top-right panel of each figure. The distribution of detected ammonia for each measurement is shown in the bottom-right panel of each figure.

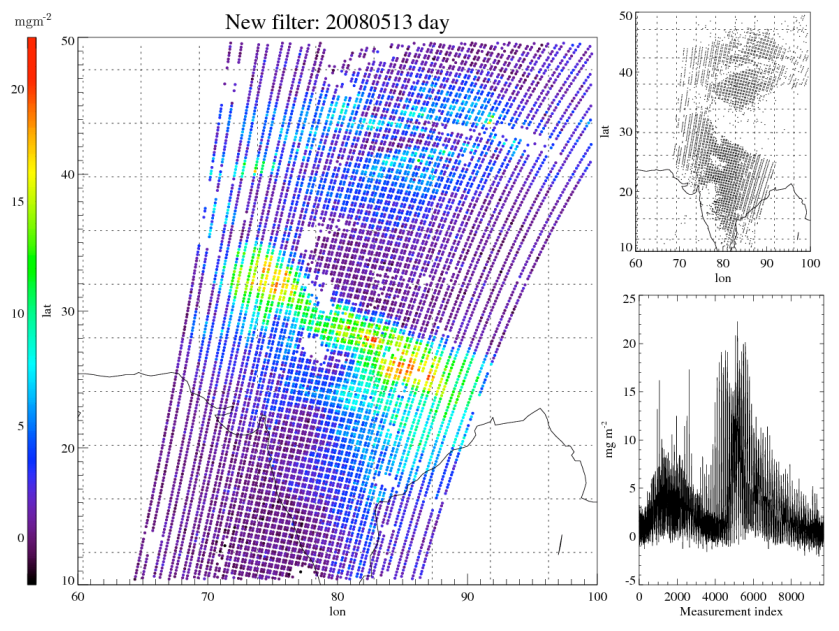

Fig. 11. As in Fig. 10 but using using the new filter for ammonia.

ammonia due to intensive agricultural practices, as identified by Clarisse et al. (2009). Cloudy pixels were removed using the method by Hadji-Lazaro et al. (2001) to ensure that any areas of high ammonia which may have appeared low due to being hidden by cloud were not included. Figures 10 and 11 show ammonia as seen by the 3 channel filter and new filter respectively for the morning overpass on the 13 May 2008. An estimated total column amount has also been derived in this case for the 3 channel ammonia filter. This estimate was derived by calculating a set of weights for the 3 channels using the same methodology as for the many channel filter, rather than using the simple 50-50 weighting between target and background channels. Error analyses
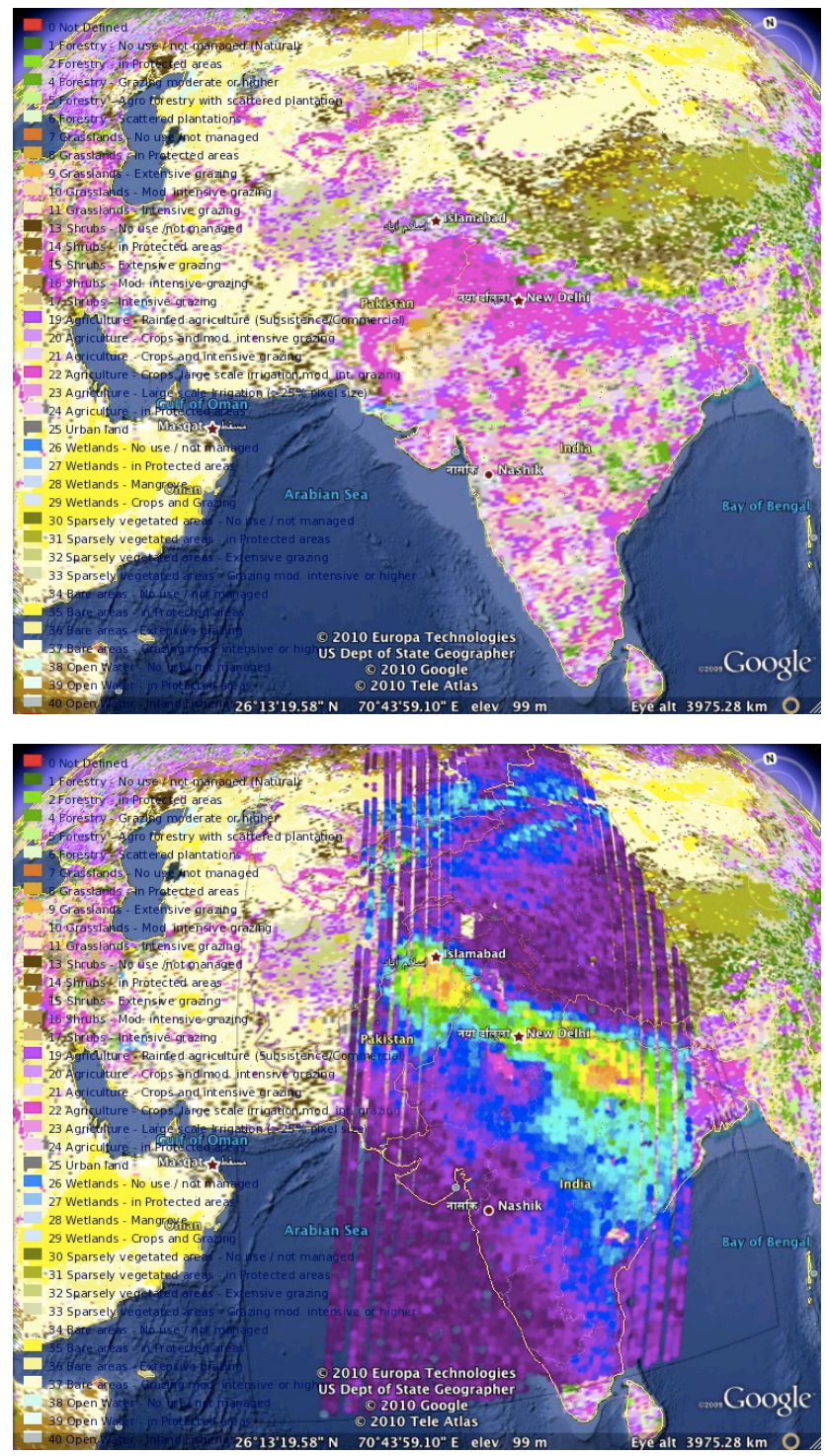

Fig. 12. Ammonia as seen by IASI across Asia for the morning overpass on 13 May 2008 using the new filter. The top panel shows a map of landuse, where pink and purple colours correspond to agriculture, green colours correspond to sparsely vegetated or forested areas, and yellow colours correspond to bare areas. Bottom panel shows ammonia overlaid onto the landuse map. Individual IASI pixels are larger than true scale.

performed for the new filter and three channel filter indicate that the detection error for the new filter is improved by over a factor 8 in comparison to the three channel filter, mainly due to the reduction in random noise. This implies that the sensitivity achieved by the many channel filter corresponds to averaging more than 64 orbits using the existing filter. The number of positive detections in the $99 \%$ confidence interval $\left(Z_{\text {thresh }}=2.725\right)$ was much higher for the new filter. Figure 12 shows a map of landuse derived from the FAOSTAT database for the year 2008 (Food and Agriculture 
Organization of the United Nations, 2008) overlaid with the ammonia field generated by the new filter to highlight the correspondence between areas of high ammonia and intensively farmed regions. Particularly high amounts of ammonia are found over an intensively cultivated region of India and Pakistan known as the Indo-Gangetic Plain. In Fig. 10, the three channel filter picks out the strongest areas of emission across the Indo-Gangetic Plain, as well as some areas of high ammonia further north. However, much more detail is visible using the new filter along the Indo-Gangetic Plain in Fig. 11, with areas of high ammonia further south across India and transport out into the Indian Ocean also visible. There also appears to be a sharp boundary corresponding to the beginning of the Himalaya mountains where ammonia concentrations are expected to be lower. Using the new filter, individual areas of agricultural production can be discerned further north with an area of high ammonia in the Fergana Valley near Tashkent, in Uzbekistan (in the area around $41^{\circ} \mathrm{N}$ $74^{\circ} \mathrm{E}$ ), which was identified as a strong source by Clarisse et al. (2009) in their yearly averaged ammonia emissions for 2008, attributed to the pooling of agricultural emissions in stangnant air. Also visible are areas of high ammonia further to the north and east, north of Tian Shan Mountain Range, Dzungaria, which were also identified as ammonia hotspots. In Fig. 12, these areas can be seen to correspond to intensively farmed regions. Tests performed on the ammonia filter using RTTOV simulated IASI spectra for a range of desert surfaces with realistic emissivities (E. Pavelin, Met Office, personal communication, 2010), did not indicate any false detections over arid regions, and so more diffuse areas of ammonia to the south-east of these agricultural valleys over arid areas could be due to the transport of polluted air over the Taklamakan desert by the prevailing winds in this region. Very little ammonia is detected in the high altitude areas of the Himalayas and Tian Shan Mountain Range, which are mostly uncultivated. Levels of ammonia over ocean are expected to be extremely low except in the case of transport from polluted areas over land. Accordingly, there are signs of transport eastward out of India over the Bay of Bengal but no ammonia detected over the Arabian Sea to the west.

The detected total column ammonia in Figs. 10 and 11 is given in $\mathrm{mg} \mathrm{m}^{-2}$. Whether or not these values reflect the true column amount depends on the linearity of the radiative transfer, the levels of the atmosphere to which the measurement is sensitive, and the conditions of thermal contrast with the surface. In the case of ammonia, the typical profile has a maximum in the boundary layer which quickly decays with altitude. The atmosphere is optically thin in this region and ammonia is weakly absorbing so radiative transfer can be assumed to be linear. These characteristics mean that it is reasonable to produce a conversion factor from the observed BTD to the estimated true column amount as in Clarisse et al. (2009). This species is therefore also a suitable candidate for producing an estimate of the true abundance using the method described in this paper. However, the apparent

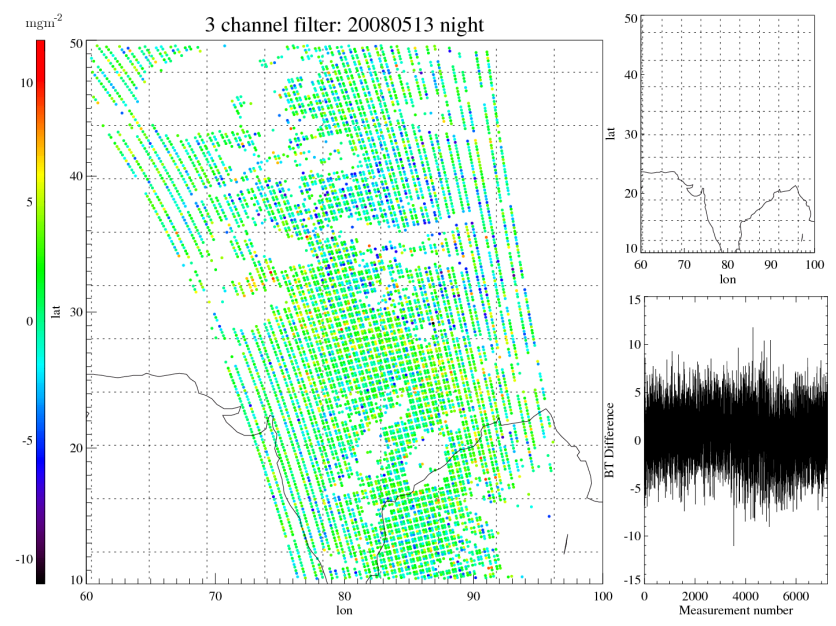

Fig. 13. Ammonia as seen by IASI for the nighttime overpass on 13 May 2008 using the exising three channel filter with estimated total column amounts given in $\mathrm{mg} \mathrm{m}^{-2}$. A map of positive detections in the $99 \%$ confidence interval is shown in the top-right panel of each figure. The distribution of detected ammonia for each measurement is shown in the bottom-right panel.

signal strength is strongly dependent on the thermal contrast between the first atmospheric layers and the surface, and the column amounts for daytime ammonia are several times higher than the total column values retrieved by Clarisse et al. (2009) using a full optimal estimation retrieval taking into account the thermal contrast conditions for this scene. Their retrieved values for this region reached $7.5 \mathrm{mg} \mathrm{m}^{-2}$ across the Indo-Gangetic Plain for this orbit, whereas the maximum estimated column values for the new filter are above $20 \mathrm{mg} \mathrm{m}^{-2}$ in this region. Favorable thermal contrast during the day makes the detection of ammonia easier but does mean that estimating the true amount would be more difficult because it depends on a knowledge of the thermal contrast conditions between the surface and the first atmospheric layers. It is possible, however, that this effect could be accounted for by using ECMWF information about the thermal contrast conditions to derive a scaling factor for the measured column so that an estimate of the abundance could be derived.

The new filter is sufficiently sensitive to detect ammonia at night when the surface temperature is often similar to the lower atmospheric layers. The results for the nighttime overpass on the 13 May 2008 are shown in Figs. 13 and 14. The $99 \%$ confidence threshold for a positive detection identifies a swath of high values in this region, as well as in several regions further north using the new filter, whereas no ammonia is positively identified using this threshold with the three channel filter. The new filter estimates column amounts for $\mathrm{NH}_{3}$ of up to $6.8 \mathrm{mg} \mathrm{m}^{-2}$, which is much lower than the estimated column value during the day, and more closely agrees with the values derived by Clarisse et al. (2009) in a full-retrieval. The nighttime spectra correspond better to 


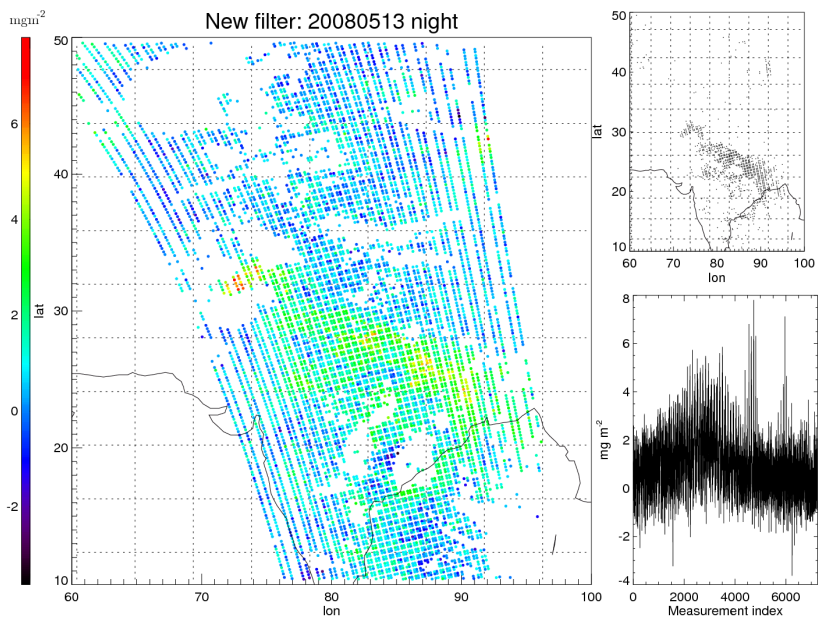

Fig. 14. As in Fig. 13 but using the new ammonia filter.

the assumption of zero thermal contrast used to generate the ammonia filter and so more realistic values can be expected. However, due to the lack of thermal contrast and therefore sensitivity to the boundary layer, it is likely that very low altitude ammonia is missed at night.

\section{Conclusions}

An extension of the brightness temperature difference method for the detection of trace species was presented and demonstrated for the detection of sulphur dioxide and ammonia from MetOp IASI. The method allows the construction of many channel filters with a set of optimal weights for the detection of the target species and suppression of other unwanted parameters. The method is based on a one-step joint retrieval of the target species and a brightness temperature offset using the total measurement error covariance to weight the least-squares inversion. The total measurement error covariance is key to the suppression of unwanted parameters in the detection. This matrix can be constructed either through consideration of appropriate perturbations to the forward model, or by using an appropriate ensemble of measured spectra to construct an estimate of the total measurement error covariance for the background atmosphere. Filters with very good signal to noise characteristics can be produced using these methods which effectively suppress the contribution of unwanted parameters, and the sensitivity of the detection of sulphur dioxide and ammonia was up to an order of magnitude more sensitive than for existing BTD filters. The method may be useful in scanning large datasets quickly for an event of interest for further quantitative analysis, or for applications such as hazard avoidance where near-real-time measurements are needed. In limited circumstances where radiative transfer is linear, there sensitivity to the entire column, and where the thermal contrast conditions with the surface can be estimated, it may be possible to perform a quantitative estimate of the total column amount. To judge whether this is possible for ammonia further work would be needed and the detected column would need to be compared against results from a full retrieval. The method should be equally applicable to other spectrometers such as AIRS-Aqua, TES-Aura, MIPAS-ENVISAT or the planned GMES Sentinel missions. The method is easy to use and could help in the provision of near-real-time monitoring of atmospheric chemistry.

Acknowledgements. This work was funded by the NERC National Centre for Earth Observation. The authors would like to thank the two anonymous reviewers for their valuable comments.

Edited by: H. Worden

\section{References}

Cayla, F., Tournier, B., and Hebert, P.: Performance budgets of IASI options, Tech. rep., Centre National d'Études Spatiales, 1995.

Clarisse, L., Coheur, P. F., Prata, A. J., Hurtmans, D., Razavi, A., Phulpin, T., Hadji-Lazaro, J., and Clerbaux, C.: Tracking and quantifying volcanic $\mathrm{SO}_{2}$ with IASI, the September 2007 eruption at Jebel at Tair, Atmos. Chem. Phys., 8, 7723-7734, doi:10.5194/acp-8-7723-2008, 2008.

Clarisse, L., Clerbaux, C., Dentener, F., Hurtmans, D., and Coheur, P.: Global ammonia distribution derived from infrared satellite observations, Nat. Geosci., 2, 479-483, 2009.

Dudhia, A.: Reference Forward Model (RFM) [Internet], http: //www.atm.ox.ac.uk/RFM (last access: October 2010), 2008.

Food and Agriculture Organization of the United Nations: FAOSTAT landuse database, [Internet], http://faostat.fao.org/site/377/ default.aspx\#ancor (last access: October 2010), 2008.

Guffanti, M., Schneider, D., Ewert, J., and Targosz, S.: Impact on aviation operations of volcanic gas and ash clouds from the 2008 eruptions of Okmok and Kasatochi, Alaska, in: AGU Fall Meeting Abstracts, vol. 1, 0277 p., 2008.

Hadji-Lazaro, J., Clerbaux, C., Couvert, P., Chazette, P., and Boonne, C.: Cloud filter for CO retrieval from IMG infrared spectra using ECMWF temperatures and POLDER cloud data, Geophys. Res. Lett., 28, 2397-2400, 2001.

Karagulian, F., Clarisse, L., Clerbaux, C., Prata, A. J., Hurtmans, D., and Coheur, P. F.: Detection of volcanic $\mathrm{SO}_{2}$, ash, and $\mathrm{H}_{2} \mathrm{SO}_{4}$ using the Infrared Atmospheric Sounding Interferometer (IASI), J. Geophys. Res., 115, D00L02, doi:10.1029/2009JD013556, 2010.

OMI Volcanic Emissions Group TOMS: AIRS Volcanic Image Archive, [Internet], http://toms.umbc.edu/, last access: October, 2010.

OMI/Aura [Internet]: http://earthobservatory.nasa.gov/IOTD/view. php?id=8998, last access: October, 2010.

Prata, A.: Satellite detection of hazardous volcanic clouds and the risk to global air traffic, Nat. Hazards, 51, 303-324, 2009.

Remedios, J. J., Leigh, R. J., Waterfall, A. M., Moore, D. P., Sembhi, H., Parkes, I., Greenhough, J., Chipperfield, M. P., and Hauglustaine, D.: MIPAS reference atmospheres and comparisons to V4.61/V4.62 MIPAS level 2 geophysical 
data sets, Atmos. Chem. Phys. Discuss., 7, 9973-10017, doi:10.5194/acpd-7-9973-2007, 2007.

Rix, M., Valks, P., Hao, N., van Geffen, J., Clerbaux, C., Clarisse, L., and Coheur, P. F.: Satellite Monitoring of Volcanic Sulfur Dioxide Emissions for Early Warning of Volcanic Hazards, IEEE J. Sel. Top. Appl., 2, 196-206, 2009.

Rodgers, C. D.: Inverse Methods for Atmospheric Sounding, World Scientific, 2000.

Rothman, L. S., Gordon, I. E., Barbe, A., Benner, D. C., Bernath, P. F., Birk, M., Boudon, V., Brown, L. R., Campargue, A., Champion, J.-P., Chance, K., Coudert, L. H., Dana, V., Devi, V. M., Fally, S., Flaud, J.-M., Gamache, R. R., Goldman, A., Jacquemart, D., Kleiner, I., Lacome, N., Lafferty, W. J., Mandin, J.-Y., Massie, S. T., Mikhailenko, S. N., Miller, C. E., Moazzen-Ahmadi, N., Naumenko, O. V., Nikitin, A. V., Orphal, J., Perevalov, V. I., Perrin, A., Predoi-Cross, A., Rinsland, C. P., Rotger, M., Šimečková, M., Smith, M. A. H., Sung, K., Tashkun, S. A., Tennyson, J., Toth, R. A., Vandaele, A. C., and Vander Auwera, J.: The HITRAN 2008 molecular spectroscopic database, J. Quantit. Spectrosc. Ra., 110, 533-572, 2009.
Sutton, M., Reis, S., and Baker, S.: Atmospheric Ammonia; Detecting emission changes and environmental impacts, Results of an Expert Workshop under the Convention on Long-range Transboundary Air Pollution, Springer, 2009.

von Clarmann, T., Grabowski, U., and Kiefer, M.: On the role of non-random errors in inverse problems in radiative transfer and other applications, J. Quant. Spectros. Ra., 71, 39-46, 2001.

van Geffen, J., Van Roozendael, M., van Gent, J., Valks, P., and Rix, M.: An alert system for volcanic $\mathrm{SO}_{2}$ emissions using satellite measurements, EUMETSAT tech rep, http://www.eumetsat.int/idcplg?IdcService= GET_FILE\&dDocName=PDF_CONF_P55_S3_20_VANGEFFE_ V\&RevisionSelectionMethod=LatestReleased (last access: October 2010), 2009. 